\title{
Equiangular frames and generalizations of the Welch bound to dual pairs of frames
}

\author{
Christensen, Ole; Datta, Somantika; Kim, Rae Young
}

Published in:

Linear and Multilinear Algebra

Link to article, DOI:

10.1080/03081087.2019.1586825

Publication date:

2019

Document Version

Peer reviewed version

Link back to DTU Orbit

Citation (APA):

Christensen, O., Datta, S., \& Kim, R. Y. (2019). Equiangular frames and generalizations of the Welch bound to dual pairs of frames. Linear and Multilinear Algebra, 1-11. https://doi.org/10.1080/03081087.2019.1586825

\section{General rights}

Copyright and moral rights for the publications made accessible in the public portal are retained by the authors and/or other copyright owners and it is a condition of accessing publications that users recognise and abide by the legal requirements associated with these rights.

- Users may download and print one copy of any publication from the public portal for the purpose of private study or research.

- You may not further distribute the material or use it for any profit-making activity or commercial gain

- You may freely distribute the URL identifying the publication in the public portal 


\title{
Equiangular frames and generalizations of the Welch bound to dual pairs of frames
}

\author{
September 19, 2018
}

\begin{abstract}
The purpose of this paper is twofold. First, we determine the lower bound for the maximum coherence between a pair of dual frames in $\mathbb{C}^{d}$ and state conditions under which the lower bound is attained. It is shown that the existence of frames and duals that attain the lower bound is related to the existence of equiangular tight frames (ETFs). Second, motivated by the scarcity of ETFs (which by default have dual ETFs), we examine the more general question of existence of equiangular frames that have equiangular duals. For the case where an equiangular dual cannot be found we provide conditions under which the number of angles among vectors in the canonical dual frame is small.
\end{abstract}

Keywords: Dual frames, equiangular frames, tight frames, Welch bound 2010 Mathematics Subject Classifications: 42C15

\section{Introduction}

Given a set $\left\{f_{i}\right\}_{i=1}^{m}$ of $m$ unit vectors in $\mathbb{C}^{d}$ with $m>d$, the lower bound on the maximum coherence between distinct vectors is given by

$$
\max _{i \neq j}\left|\left\langle f_{i}, f_{j}\right\rangle\right|^{2} \geq \frac{m-d}{d(m-1)} .
$$

The quantity on the right of (1.1) is known as the Welch bound [16]. Sets that attain the lower bound in (1.1), often called Welch bound equality sets or equiangular tight frames (ETFs), arise in various application areas such as communication systems, quantum information processing, and coding theory $[9,11,10]$. Consequently, the problem of constructing ETFs and determining conditions under which they exist has gained substantial attention $[11,12,8,1]$.

In the literature one finds extensions and generalizations of the Welch bound to infinite dimensional spaces [11], to generalized or continuous frames [5], and to correlation between 
subspaces [4]. In the work presented here generalizations of the Welch bound in (1.1) are considered that are two-fold in nature. In Section 2 , fixing some $m \in \mathbb{N}$, we consider dual pairs of frames $\left\{f_{i}\right\}_{i=1}^{m},\left\{g_{i}\right\}_{i=1}^{m}$ for $\mathbb{C}^{d}$ and determine a lower bound on $\max _{i \neq j}\left|\left\langle f_{i}, g_{j}\right\rangle\right|^{2}$. Conditions under which the lower bound is attained, and pairs of sets that attain the lower bound are characterized. We also prove that the existence of frames and duals that attain the lower bound is equivalent to the existence of an ETF of $m$ vectors in $\mathbb{C}^{d}$. Then, in Section 3, we generalize the theory of sets that attain the lower bound in (1.1) by analyzing equiangular frames that are not necessarily tight and their duals. As indicated above, the focus in the literature has been on equiangular tight frames, and these already have equiangular duals. Unfortunately, for many pairs $(m, d)$, ETFs do not exist [12], and even when they exist they are hard to construct. Here we consider equiangular frames that are not necessarily tight and investigate whether they can have an equiangular dual. When an equiangular dual cannot be found, conditions are given under which an equiangular frame has a dual such that the number of distinct angles among the dual frame vectors is small.

In the rest of this introduction we collect some definitions and known results that will be used throughout the paper. In a finite dimensional Hilbert space like $\mathbb{R}^{d}$ or $\mathbb{C}^{d}$, a frame consisting of a finite number of vectors is the same as a spanning set. Given a set $\Phi=\left\{f_{1}, \ldots, f_{m}\right\}$ in $\mathbb{R}^{d}$ or $\mathbb{C}^{d}$, let $T$ be the matrix whose columns are the vectors $f_{1}, \ldots, f_{m}$. $T$ is called the synthesis operator of $\Phi$. If $\Phi$ is a frame then the $d \times d$ matrix $T T^{*}$ is called the frame operator of $F$. The set $\Phi$ is said to be a tight frame if the frame operator is a constant multiple of the identity. The matrix $T^{*} T$ is the Gram matrix of the set $\Phi$. The $(i, j)$ th entry of the Gram matrix is the inner product $\left\langle f_{j}, f_{i}\right\rangle$. A frame of $m$ vectors in a $d$-dimensional space will be referred to as an $(m, d)$ frame.

Definition 1.1 [12] An equiangular tight frame (ETF) is a set $\left\{f_{i}\right\}_{i=1}^{m}$ in a d-dimensional Hilbert space satisfying

(i) $T T^{*}=\frac{m}{d} I$, i.e., the set is a tight frame.

(ii) $\left\|f_{i}\right\|=1$, for $i=1, \ldots, m$, i.e., the set is unit normed.

(iii) $\left|\left\langle f_{i}, f_{j}\right\rangle\right|=\sqrt{\frac{m-d}{d(m-1)}}, 1 \leq i \neq j \leq m$, i.e., the set meets the Welch bound.

Relaxing the condition of being tight in Definition 1.1 gives an equiangular frame.

Definition 1.2 [15] An equiangular frame (EF) is a set $\left\{f_{i}\right\}_{i=1}^{m}$ in a d-dimensional Hilbert space $\mathcal{H}$ that is a frame for $\mathcal{H}$ and satisfies the following for some $\alpha>0$.

(i) $\left\|f_{i}\right\|=1$, for $i=1, \ldots, m$.

(ii) $\left|\left\langle f_{i}, f_{j}\right\rangle\right|=\alpha, 1 \leq i \neq j \leq m$.

Note that in Definition 1.2, $\alpha$ equals the Welch bound only when the set is a tight frame. For an equiangular frame, the off-diagonal entries of the associated Gram matrix all have modulus equal to $\alpha$. Generalizing this one gets the following. 
Definition 1.3 A frame $\left\{f_{i}\right\}_{i=1}^{m}$ for a d-dimensional Hilbert space $\mathcal{H}$ is called a k-angle frame or a frame with $k$-angles if the associated Gram matrix has off-diagonal entries with $k$ distinct moduli. The number of distinct values taken by the set $\left\{\left|\left\langle f_{i}, f_{j}\right\rangle\right|\right\}_{1 \leq i<j \leq m}$ is called the number of angles of the frame.

The Gram matrix of an EF of $m$ vectors in a $d$-dimensional space can be written as

$$
G=I+\alpha Q
$$

where $Q$ is an $m \times m$ Hermitian matrix with zero diagonal and unimodular entries elsewhere, called the signature matrix. The Gram matrix of $\Phi$ will have a zero eigenvalue of multiplicity $m-d$. This implies that the minimum eigenvalue of $Q$ is $-1 / \alpha$ with multiplicity $m-d$ [8]. This property is used later in Section 3. Due to (1.2), the study of EFs reduces to the study of the corresponding Gram matrices or signature matrices. In this paper, all the results pertain to real EFs.

\section{Equiangular Dual Frames}

In this section we derive generalizations of the Welch bounds for tight frames to dual pairs of frames. We will repeatedly need the following result, which is Theorem 3.1 in [3].

Lemma 2.1 Given $\left\{\alpha_{i}\right\}_{i=1}^{m} \subset \mathbb{C}$, the following are equivalent:

(a) There exist dual frames $\left\{f_{i}\right\}_{i=1}^{m}$ and $\left\{g_{i}\right\}_{i=1}^{m}$ for $\mathbb{C}^{d}$ such that $\alpha_{i}=\left\langle f_{i}, g_{i}\right\rangle$ for all $1 \leq i \leq m$;

(b) $d=\sum_{i=1}^{m} \alpha_{i}$.

Theorem 2.2 Let $\left\{f_{i}\right\}_{i=1}^{m}$ be a frame for $\mathbb{C}^{d}$ with frame operator $S$, and let $\left\{g_{i}\right\}_{i=1}^{m}$ denote a dual frame of $\left\{f_{i}\right\}_{i=1}^{m}$. Then

$$
\sum_{i=1}^{m} \sum_{j=1}^{m}\left|\left\langle f_{i}, g_{j}\right\rangle\right|^{2} \geq d
$$

Equality holds if and only if $g_{i}=S^{-1} f_{i}$ for $i=1, \cdots, m$.

Proof. Considering the expansion of any $f \in \mathbb{C}^{d}$ in terms of the frame $\left\{f_{i}\right\}_{i=1}^{m}$, it is wellknown (see [2, Lemma 5.4.2]) that the $\ell^{2}$-norm of the coefficients is minmimized precisely for the canonical frame coefficients, i.e., for $i \in\{1, \cdots, m\}$ we have

$$
\sum_{j=1}^{m}\left|\left\langle f_{i}, g_{j}\right\rangle\right|^{2} \geq \sum_{j=1}^{m}\left|\left\langle f_{i}, S^{-1} f_{j}\right\rangle\right|^{2}
$$

where equality holds if and only if $g_{i}=S^{-1} f_{i}, i=1, \cdots, m$. Note that $\left\{S^{-1 / 2} f_{j}\right\}_{j=1}^{m}$ is a tight frame with frame bound 1 . Then we have $\sum_{i=1}^{m}\left\|S^{-1 / 2} f_{i}\right\|^{2}=d$ by Lemma 2.1 and so

$$
\sum_{i=1}^{m} \sum_{j=1}^{m}\left|\left\langle f_{i}, S^{-1} f_{j}\right\rangle\right|^{2}=\sum_{i=1}^{m} \sum_{j=1}^{m}\left|\left\langle S^{-1 / 2} f_{i}, S^{-1 / 2} f_{j}\right\rangle\right|^{2}=\sum_{i=1}^{m}\left\|S^{-1 / 2} f_{i}\right\|^{2}=d .
$$


This together with (2.1) yields the result.

Theorem 2.3 Let $\left\{f_{i}\right\}_{i=1}^{m}$ be a frame for $\mathbb{C}^{d}$, and let $\left\{g_{i}\right\}_{i=1}^{m}$ denote a dual frame of $\left\{f_{i}\right\}_{i=1}^{m}$. Assume that $\left\langle f_{i}, g_{i}\right\rangle$ is constant for $i=1, \cdots, m$. Then

$$
\max _{i \neq j}\left|\left\langle f_{i}, g_{j}\right\rangle\right|^{2} \geq \frac{d(m-d)}{m^{2}(m-1)} .
$$

Equality holds if and only if $g_{i}=S^{-1} f_{i}$ for $i=1, \cdots, m$ and $\left|\left\langle f_{i}, g_{j}\right\rangle\right|$ is constant for $i \neq j$.

Proof. First we note that since $\left\langle f_{j}, g_{j}\right\rangle$ is constant for $j=1, \cdots, m$ and $\sum_{i=1}^{m}\left\langle f_{i}, g_{i}\right\rangle=d$ by Lemma 2.1, we have

$$
\sum_{i=1}^{m}\left|\left\langle f_{i}, g_{i}\right\rangle\right|^{2}=\sum_{i=1}^{m}\left|\frac{1}{m} \sum_{j=1}^{m}\left\langle f_{j}, g_{j}\right\rangle\right|^{2}=\frac{d^{2}}{m} .
$$

Now using Theorem 2.2 and (2.4), we have

$$
\begin{aligned}
\max _{i \neq j}\left|\left\langle f_{i}, g_{j}\right\rangle\right|^{2} & \geq \frac{1}{m(m-1)} \sum_{i \neq j}\left|\left\langle f_{i}, g_{j}\right\rangle\right|^{2} \\
& =\frac{1}{m(m-1)}\left(\sum_{i=1}^{m} \sum_{j=1}^{m}\left|\left\langle f_{i}, g_{j}\right\rangle\right|^{2}-\sum_{k=1}^{m}\left|\left\langle f_{k}, g_{k}\right\rangle\right|^{2}\right) \\
& \geq \frac{1}{m(m-1)}\left(d-\frac{d^{2}}{m}\right)=\frac{d(m-d)}{m^{2}(m-1)} .
\end{aligned}
$$

By Theorem 2.2 again, equality is attained in the third step if and only if $g_{i}=S^{-1} f_{i}$ for $i=1, \cdots, m$. Then equality is attained in the first step if and only if $\left|\left\langle f_{i}, g_{j}\right\rangle\right|$ is constant for $i \neq j$.

Proposition 2.4 Under the assumptions in Theorem 2.3, the following are equivalent:

(1) Equality holds in (2.3);

(2) The associated mixed Gramian can be written as

$$
G=\frac{d}{m}(I+\alpha Q)
$$

where $I$ is the identity martix, $\alpha:=\sqrt{\frac{m-d}{d(m-1)}}$, and $Q$ is a Hermitian matrix with zeros along the diagonal and unimodular entries elsewhere.

(3) An $(m, d)$ ETF exists.

Proof. $\quad(1) \Leftrightarrow(2)$ This follows from Lemma 2.1 and Theorem 2.3.

$(2) \Leftrightarrow(3)$ This follows from the fact that $I+\alpha Q$ with $\alpha=\sqrt{\frac{m-d}{d(m-1)}}$ is the Gramian of an $(m, d) \mathrm{ETF}$. 


\section{Equiangular frames and their duals}

Phrased in terms of Definition 1.3, an ETF is an 1-angle frame such that the canonical dual is also an 1-angle frame. However, it is well-known that for many pairs $(m, d)$, ETFs do not exist [12]. In this section we investigate conditions under which an equiangular frame that is not tight can have a dual that is a $k$-angle frame where it is desired that $k$ is a small positive integer. Due to (1.2), the study of EFs reduces to the study of the corresponding signature matrices. Signature matrices with two distinct eigenvalues correspond to ETFs. Examples of such signature matrices are somewhat rare $[12,13]$, as are ETFs. It is known that various large sets of equiangular lines have corresponding signature matrices with three distinct eigenvalues [7]. This has motivated extensive study of signature matrices with exactly three eigenvalues in $[7,13]$. In Theorem 3.3 and Theorem 3.5 below, we analyze signature matrices with three distinct eigenvalues and study the number of possible angles in the canonical dual of the corresponding equiangular frame. It is worth noting that a result similar to Theorem 3.3 using strongly regular graphs is given in [15]. Note that since a signature matrix has zero trace, the minimum eigenvalue must be negative. In accordance with the notation in existing literature [15], the minimum eigenvalue is written as $-\lambda_{1}$ where $\lambda_{1}$ is positive. The value of $\alpha$ in (1.2) is $1 / \lambda_{1}$. The following Lemma 3.1 will be used.

Lemma 3.1 If $-\lambda_{1}, \lambda_{2}, \lambda_{3}$ are the three distinct eigenvalues of a signature matrix $Q$, ordered such that $-\lambda_{1}<\lambda_{2}<\lambda_{3}, \lambda_{1}>0$, then

$$
-\lambda_{1} \neq \frac{\lambda_{2}+\lambda_{3}}{2}
$$

Proof. Since $Q$ has zero trace, it must have at least one positive eigenvalue, so $\lambda_{3}>0$. Now, if $-\lambda_{1}=\frac{\lambda_{2}+\lambda_{3}}{2}$, then $\lambda_{2}+\lambda_{3}<0$; thus $-\lambda_{1}<\lambda_{2}<\lambda_{2}+\lambda_{3}<0$, which is a contradiction.

The following lemma can be proved by a direct calculation of the characteristic polynomial of $Q$.

Lemma 3.2 If $Q$ is an $m \times m$ signature matrix whose off-diagonal entries are all 1 then $Q$ has two distinct eigenvalues: $m-1$ with multiplicity 1 , and -1 with multiplicity $m-1$.

An eigenvector is said to be regular if its entries are \pm 1 . In what follows, $\overrightarrow{1}$ denotes the vector whose each entry is 1 , and $J$ is the matrix whose entries are all 1 . The $(i, j)$ th entry of a matrix $A$ will be denoted by $A(i, j)$.

Theorem 3.3 Let $Q$ be an $m \times m$ signature matrix with three distinct eigenvalues $-\lambda_{1}, \lambda_{2}, \lambda_{3}$, ordered such that $-\lambda_{1}<\lambda_{2}<\lambda_{3}$, with $\lambda_{1}>0$. Let $\Phi$ denote the corresponding equiangular frame of $m$ vectors in $\mathbb{R}^{d}$. Then the following hold.

(a) Suppose that $\lambda_{2}$ or $\lambda_{3}$ is a simple eigenvalue with a regular eigenvector, and the multiplicity of $-\lambda_{1}$ is $\mu$. Then the canonical dual is an equal norm 2-angle non-tight frame and $d=m-\mu$. 
(b) Suppose that the minimum eigenvalue $-\lambda_{1}$ is simple with a regular eigenvector. If the eigenvector is $\overrightarrow{1}$, then the canonical dual is an equal norm 2-angle non-tight frame. Otherwise, the canonical dual is an equal norm $k$-angle non-tight frame where $2 \leq k \leq 4$. In this case, $d=m-1$.

Proof. The Gram matrix of a tight frame can have only one non-zero eigenvalue. Since $Q$ has three distinct eigenvalues, $G$ must have two distinct nonzero eigenvalues and so $\Phi$ is a not a tight frame. Thus the dual is also not tight in both (a) and (b).

Let $P_{1}, P_{2}$, and $P_{3}$ denote the orthogonal projections onto the eigenspaces of $-\lambda_{1}$, $\lambda_{2}$, and $\lambda_{3}$, respectively. By the Spectral Theorem $Q=-\lambda_{1} P_{1}+\lambda_{2} P_{2}+\lambda_{3} P_{3}$. where $P_{1}+P_{2}+P_{3}=I$, and for $i \neq j, P_{i} P_{j}=0$. The Gram matrix of $\Phi$ is

$$
G=I+\frac{1}{\lambda_{1}} Q=\frac{\lambda_{1}+\lambda_{2}}{\lambda_{1}} P_{2}+\frac{\lambda_{1}+\lambda_{3}}{\lambda_{1}} P_{3}
$$

The Gram matrix of the canonical dual is the pseudo inverse of $G$ [2], and given by

$$
G^{\dagger}=\frac{\lambda_{1}}{\lambda_{1}+\lambda_{2}} P_{2}+\frac{\lambda_{1}}{\lambda_{1}+\lambda_{3}} P_{3}
$$

(a) Since the multiplicity of the minimum eigenvalue of $Q$ is $\mu=m-d$, the value of $d$ is obvious. Without loss of generality assume that $\lambda_{3}$ is simple with a regular eigenvector $v$. Then $P_{3}=\frac{1}{\|v\|^{2}} v v^{\mathrm{T}}=\frac{1}{m} v v^{\mathrm{T}}$. Note that the diagonal entries of $P_{3}$ are all equal to $\frac{1}{m}$. This implies, from (3.1), that $P_{2}$ also has constant diagonal. Therefore, in (3.2), $G^{\dagger}$ must have constant diagonal too, implying that the canonical dual is equal norm.

Equating the off-diagonal entries of $G$ in (3.1) gives

$$
\pm \frac{1}{\lambda_{1}}=\frac{\lambda_{1}+\lambda_{2}}{\lambda_{1}} P_{2}(i, j)+\frac{\lambda_{1}+\lambda_{3}}{\lambda_{1}} P_{3}(i, j) .
$$

or,

$$
P_{2}(i, j)=\left[ \pm 1-\left(\lambda_{1}+\lambda_{3}\right) P_{3}(i, j)\right] \frac{1}{\lambda_{1}+\lambda_{2}}
$$

Using (3.2), (3.3), and the fact that the off-diagonal entries of $P_{3}$ are $\pm \frac{1}{m}$

$$
G^{\dagger}(i, j)= \pm \frac{\lambda_{1}}{\left(\lambda_{1}+\lambda_{2}\right)^{2}}+\left( \pm \frac{1}{m}\right)\left[\frac{\lambda_{1}}{\lambda_{1}+\lambda_{3}}-\frac{\lambda_{1}\left(\lambda_{1}+\lambda_{3}\right)}{\left(\lambda_{1}+\lambda_{2}\right)^{2}}\right], \quad i \neq j .
$$

From (3.4), the absolute values of the off-diagonal entries of $G^{\dagger}$ can take only two values, and this can be justified as follows. The expression inside the bracket in the second term on the right side of (3.4) cannot be zero due to Lemma 3.1. Since $Q$ has three distinct eigenvalues, $Q$ cannot cannot have all its off-diagonal entries equal to 1 or all equal to -1 due to Lemma 3.2. Thus the off-diagonal entries of $G$ take both values $\pm \frac{1}{\lambda_{1}}$. If $P_{3}$ and $G$ have the exact same or the exact opposite sign distribution in their off-diagonal entries, then, from (3.3), the off-diagonal entries of $P_{2}$ will equal $\pm c$ for some constant $c$ and have 
the same sign distribution as that of $P_{3}$ or $G$. In that case, $P_{2} P_{3} \neq 0$, which contradicts the Spectral Theorem. Thus $P_{3}$ and $G$ cannot have the exact same or the exact opposite sign distribution. All this suggests that the absolute values of the off-diagonal entries of $G^{\dagger}$ take only two values, and the canonical dual is a 2-angle frame.

(b) Suppose that $-\lambda_{1}$ is a simple eigenvalue. Since the multiplicity of the minimum eigenvalue is 1 , the frame is in $\mathbb{R}^{m-1}$.

If $\overrightarrow{1}$ is an eigenvector for $-\lambda_{1}$ then $Q=-\lambda_{1} \frac{J}{m}+\lambda_{2} P_{2}+\lambda_{3} P_{3}$. Using the fact that $\frac{J}{m}+P_{2}+P_{3}=I$ in $(3.1)$ gives

$$
G=\frac{\lambda_{1}+\lambda_{2}}{\lambda_{1}} I-\frac{\lambda_{1}+\lambda_{2}}{\lambda_{1}} \frac{J}{m}+\frac{\lambda_{3}-\lambda_{2}}{\lambda_{1}} P_{3} .
$$

The Gram matrix of the dual then becomes

$$
G^{\dagger}=\frac{\lambda_{1}}{\lambda_{1}+\lambda_{2}} P_{2}+\frac{\lambda_{1}}{\lambda_{1}+\lambda_{3}} P_{3}=\frac{\lambda_{1}}{\lambda_{1}+\lambda_{2}}\left(I-\frac{J}{m}\right)+\lambda_{1}\left(\frac{1}{\lambda_{1}+\lambda_{3}}-\frac{1}{\lambda_{1}+\lambda_{2}}\right) P_{3} .
$$

Equating the diagonal and off-diagonal entries of $G$ and $G^{\dagger}$, one can conclude that in this case the dual is 2-angle and equal norm.

Next suppose that $-\lambda_{1}$ is a simple eigenvalue with a regular eigenvector $v$ that is not $\overrightarrow{1}$. This time

$$
G=\frac{\lambda_{1}+\lambda_{2}}{\lambda_{1}} I-\frac{\lambda_{1}+\lambda_{2}}{\lambda_{1}} P_{1}+\frac{\lambda_{3}-\lambda_{2}}{\lambda_{1}} P_{3}
$$

and

$$
G^{\dagger}=\frac{\lambda_{1}}{\lambda_{1}+\lambda_{2}} I-\frac{\lambda_{1}}{\lambda_{1}+\lambda_{2}} P_{1}+\frac{\lambda_{1}\left(\lambda_{2}-\lambda_{3}\right)}{\left(\lambda_{1}+\lambda_{3}\right)\left(\lambda_{1}+\lambda_{2}\right)} P_{3} .
$$

Note that $P_{1}=\frac{1}{m} v v^{\mathrm{T}}$ with diagonal entries all equal to $\frac{1}{m}$. Thus, in (3.5), the matrices $G, I$, and $P_{1}$ all have constant diagonal. This implies that $P_{3}$ also has constant diagonal. Using this in (3.6) shows that $G^{\dagger}$ also has constant diagonal, i.e., the canonical dual frame is equal norm.

Solving for $P_{3}$ in (3.5), and using the fact that the off-diagonal entries of $P_{1}$ are $\pm \frac{1}{m}$, gives

$$
P_{3}(i, j)=\frac{1}{\lambda_{3}-\lambda_{2}}\left[ \pm 1 \pm \frac{\lambda_{1}+\lambda_{2}}{m}-\left(\lambda_{1}+\lambda_{2}\right)\right], \quad \text { for } i \neq j
$$

Substituting (3.7) in (3.6), gives for $i \neq j$

$$
\begin{aligned}
G^{\dagger}(i, j) & =\frac{\lambda_{1}}{\lambda_{1}+\lambda_{2}}\left[1+\frac{1}{\lambda_{1}+\lambda_{3}}\right] \mp \frac{1}{m} \lambda_{1} \frac{2 \lambda_{1}+\lambda_{2}+\lambda_{3}}{\left(\lambda_{1}+\lambda_{2}\right)\left(\lambda_{1}+\lambda_{3}\right)} \mp \frac{\lambda_{1}}{\left(\lambda_{1}+\lambda_{2}\right)\left(\lambda_{1}+\lambda_{3}\right)} \\
& =C_{1} \mp \frac{1}{m} C_{2} \mp C_{3}
\end{aligned}
$$

where $C_{1}, C_{2}$, and $C_{3}$ are distinct nonzero constants due to properties of eigenvalues of $Q$ and Lemma 3.1. Thus the dual cannot be equiangular and the absolute values of the off-diagonal entries of $G^{\dagger}$ can take up to 4 distinct values. This implies that the canonical dual is a $k$-angle frame where $2 \leq k \leq 4$. 
By $[\lambda]^{n}$ it will be meant that the eigenvalue $\lambda$ has multiplicity $n$. Suppose that a signature matrix $Q$ with three distinct eigenvalues has an irrational eigenvalue $\lambda+\sqrt{\mu}$. Then the other two eigenvalues of $Q$ are $\lambda-\sqrt{\mu}$ and some $k \in \mathbb{Z}[7,13]$. The following result proved in [6] will be used.

Lemma 3.4 (Corollary 5.6 [6] ) Let $Q$ be an $m \times m$ signature matrix with three distinct eigenvalues, at least one of which is irrational. If $m$ is odd then the eigenvalues of $Q$ are

$$
[-\sqrt{m}]^{(m-1) / 2},[0]^{1},[\sqrt{m}]^{(m-1) / 2} .
$$

Theorem 3.5 Let $Q$ be an $m \times m$ signature matrix with three distinct eigenvalues, at least one of which is irrational. Let $\Phi$ denote the corresponding equiangular frame of $m$ vectors in $\mathbb{R}^{d}$.

(i) If $m$ is odd, then the canonical dual is an equal norm frame with at most $m-1$ angles, and $d=\frac{m+1}{2}$.

(ii) Let $Q$ have eigenvalues

$$
[-k]^{m-2 n},[a-\sqrt{b}]^{n},[a+\sqrt{b}]^{n}
$$

with minimum eigenvalue $-k, k \in \mathbb{Z}^{+}, a \in \mathbb{Q}, b \in \mathbb{Q}^{+}$, and $m-2 n>1$. Let the number of distinct moduli in the irrational part of the projection matrix of the eigenspace of either $a+\sqrt{b}$ or $a-\sqrt{b}$ be $p$. Then the canonical dual has at most $2 p$ angles, and $d=2 n$.

Proof. (i) Due to Lemma 3.4, the eigenvalues of $Q$ in this case are

$$
[-\sqrt{m}]^{(m-1) / 2},[0]^{1},[\sqrt{m}]^{(m-1) / 2} .
$$

Since the multiplicity of the minimum eigenvalue is $\frac{m-1}{2}$, the value of $d$ is given by

$$
d=m-\frac{m-1}{2}=\frac{m+1}{2} .
$$

Denote the projection matrices of $-\sqrt{m}$ and $\sqrt{m}$ by $P_{1}$ and $P_{\widehat{1}}$, respectively. By the Spectral Theorem $Q=-\sqrt{m} P_{1}+\sqrt{m} P_{\widehat{1}}$. Note that $P_{1}$ and $P_{\widehat{1}}$ are irrational conjugates of each other, and can be written as $P_{1}=P_{a}+P_{b}, \quad P_{\widehat{1}}=P_{a}-P_{b}$ where the $(i, j)$ th entries of $P_{a}$ and $P_{b}$ are given by

$$
\begin{aligned}
& P_{a}(i, j)=a_{i j} \in \mathbb{Q} \\
& P_{b}(i, j)=0 \text { or } \sqrt{b_{i j}}, b_{i j} \in \mathbb{Q}, b_{i j} \text { not a perfect square. }
\end{aligned}
$$

It follows that $Q=-2 \sqrt{m} P_{b}$. The Gram matrix of $\Phi$ and the Gram matrix of the canonical dual are given by

$$
\begin{aligned}
G & =I+\frac{1}{\sqrt{m}} Q=I-P_{1}+P_{\widehat{1}}=I-2 P_{b}, \\
G^{\dagger} & =I-P_{1}-\frac{1}{2} P_{\widehat{1}}=I-\frac{3}{2} P_{a}-\frac{1}{2} P_{b} .
\end{aligned}
$$


Using the relations

$$
P_{1}^{2}=P_{1}, \quad, P_{\widehat{1}}^{2}=P_{\widehat{1}}, \quad P_{\widehat{1}} P_{1}=0, \quad P_{1} P_{\widehat{1}}=0,
$$

one gets $P_{a}=2 P_{b}^{2}$ and thus

$$
G^{\dagger}=I-3 P_{b}^{2}-\frac{1}{2} P_{b}
$$

The off-diagonal entries of $G$ are $\pm \frac{1}{\sqrt{m}}$, and its diagonal entries are all equal to 1 . Equating the $(i, j)$ th entries of the matrices in (3.8) then gives

$$
P_{b}(i, j)=\left\{\begin{array}{cc}
0 & \text { if } i=j \\
\pm \frac{1}{2 \sqrt{m}} & \text { if } i \neq j
\end{array}\right.
$$

Let $\beta:=\frac{1}{2 \sqrt{m}}$. The diagonal entries of $P_{b}^{2}$ are then all equal to $(m-1) \beta^{2}$. Since the diagonal entries of $P_{b}$ are all equal to zero, this means from (3.10) that $G^{\dagger}$ has constant diagonal, and that the canonical dual is equal norm.

The absolute values of the off-diagonal entries of $P_{b}^{2}$ can take at most $\frac{m-1}{2}$ distinct values given by

$$
\left\{(m-2) \beta^{2},(m-4) \beta^{2}, \cdots, \beta^{2}\right\} .
$$

This combined with the fact that $I-\frac{1}{2} P_{b}$ can take the values $1 \pm \frac{\beta}{2}$ means that the absolute values of the off diagonal entries of $G^{\dagger}$ can take at most $m-1$ distinct values.

(ii) Now the multiplicity of the minimum eigenvalue is $m-2 n$, and so $d$ equals $2 n$. Let $P_{2}$ and $P_{\widehat{2}}$ denote the projection matrices of $a+\sqrt{b}$ and $a-\sqrt{b}$, respectively. As in part(i), these can be written as $P_{2}=P_{a}+P_{b}, \quad P_{\widehat{2}}=P_{a}-P_{b}$, Let $P_{1}$ denote the projection matrix of $-k$. Since $m-2 n>1, \overrightarrow{1}$ is not a basis for the eigenspace of $-k$. Thus $P_{1} \neq \frac{1}{m} J$, and the number of angles in the canonical dual cannot be determined by Theorem 3.3. By the Spectral Theorem

$$
Q=-k P_{1}+(a+\sqrt{b})\left(P_{a}+P_{b}\right)+(a-\sqrt{b})\left(P_{a}-P_{b}\right) .
$$

Using $P_{1}+P_{2}+P_{\widehat{2}}=I$ gives

$$
G=I+\frac{1}{k} Q=\frac{2}{k}\left((k+a) P_{a}+\sqrt{b} P_{b}\right) .
$$

The Gram matrix of the canonical dual is the pseudo inverse

$$
G^{\dagger}=\frac{2 k}{(k+a)^{2}-b}\left[\frac{k G}{2}-2 \sqrt{b} P_{b}\right] .
$$

The result then follows from the fact that since $\Phi$ is equiangular, the off diagonal entries of $G$ are either $\frac{1}{k}$ or $-\frac{1}{k}$.

Due to the algebraic properties of signature matrices [13], one cannot expect to generalize the above results to any arbitrary number of distinct eigenvalues of $Q$. In the context of regular graphs, signature matrices with four eigenvalues are discussed in [14], and for this case, Theorem 3.5 can then be extended as follows. 
Theorem 3.6 Let $Q$ be an $m \times m$ signature matrix with four distinct eigenvalues

$$
[a-\sqrt{b}]^{n},[a+\sqrt{b}]^{n},\left[-k_{1}\right]^{m-2 n-1},\left[k_{2}\right]^{1}
$$

with minimum eigenvalue $-k_{1}$, where $k_{1}, k_{2} \in \mathbb{Z}^{+}, a \in \mathbb{Q}, b \in \mathbb{Q}^{+}$. Suppose that $\overrightarrow{1}$ is an eigenvector of $Q$ corresponding to $k_{2}$. Let the number of distinct moduli in the purely irrational part of the projection matrix of the eigenspace of either $a+\sqrt{b}$ or $a-\sqrt{b}$ be $p$. If $\Phi$ is the equiangular frame corresponding to $Q$ then the canonical dual is a frame in $\mathbb{R}^{2 n+1}$ having at most $2 p$ angles.

Proof. The projection matrix of the eigenspace of $k_{2}$ is $\frac{J}{m}$. The spectral decomposition of $Q$ is

$$
Q=-k_{1} P_{1}+k_{2} \frac{J}{m}+(a+\sqrt{b}) P_{2}+(a-\sqrt{b}) P_{\widehat{2}}
$$

The proof then follows in an identical manner as Theorem 3.5 by noting that the off diagonal entries of $J$ are all one.

\section{References}

[1] B. G. Bodmann, V. I. Paulsen, and M. Tomforde. Equiangular tight frames from complex Seidel matrices containing cube roots of unity. Linear Algebra Appl., 430(1):396417, 2009.

[2] O. Christensen, An introduction to frames and Riesz bases. Second expanded edition. Birkhäuser (2016)

[3] O. Christensen, A. Powell, and X.C. Xiao, A note on finite dual frame pairs. Proc. Amer. Math. Soc. 140 (2012), no. 11, 3921-3930.

[4] S. Datta. Welch bounds for cross correlation of subspaces and generalizations. Linear and Multilinear Algebra, 64(8):1484-1497, 2016.

[5] S. Datta, S. D. Howard, and D. Cochran. Geometry of the Welch bounds. Linear Algebra and its Applications, 437(10):2455 - 2470, 2012.

[6] G. Greaves. Equiangular lines systems and switching classes containing regular graphs. Linear Algebra Appl., in press, http://dx.doi.org/10.1016/j.laa.2017.09.008, 2017.

[7] G. Greaves and J. H. Koolen and A. Munemasa and F. Szöllösi. Equiangular lines in Euclidean spaces. Journal of Combinatorial Theory, Series A., 138: 208 - 235, 2016.

[8] R. B. Holmes and V. I. Paulsen. Optimal frames for erasures. Linear Algebra Appl., $377: 31-51,2004$. 
[9] J. L. Massey and T. Mittelholzer. Welch's bound and sequence sets for code-division multiple-access systems. In R. Capocelli, A. De Santis, and U. Vaccaro, editors, Sequences II: Methods in Communication, Security and Computer Science, pages 63-78. Springer-Verlag, New York, 1991.

[10] J. M. Renes, R. Blume-Kohout, A. J. Scott, and C. M. Caves. Symmetric informationally complete quantum measurements. Journal of Mathematical Physics, 45(6):2171 2180, 2004.

[11] T. Strohmer and R. W. Heath, Jr. Grassmannian frames with applications to coding and communication. Applied and Computational Harmonic Analysis, 14(3):257-275, 2003.

[12] M. A. Sustik, J. A. Tropp, I. S. Dhillon, and R. W. Heath, Jr. On the existence of equiangular tight frames. Linear Algebra Appl., 426(2-3):619-635, 2007.

[13] F. Szöllősi and R. J. Östergård. Enumeration of Seidel matrices. arXiv:1703.02943, 2017.

[14] E. R. van Dam. Regular graphs with four eigenvalues. Linear Algebra and its Applications. 226-228: 139 - 162, 1995.

[15] S. Waldron. On the construction of equiangular frames from graphs. Linear Algebra and its Applications, 431(11):2228-2242, 2009.

[16] L. R. Welch. Lower bounds on the maximum cross correlation of signals. IEEE Transactions on Information Theory, 20(3):397-399, May 1974.

\section{Ole Christensen}

Department of Applied Mathematics and Computer Science Technical University of Denmark, Building 303, 2800 Lyngby

Denmark, Email: ochr@dtu.dk

Somantika Datta

Department of Mathematics

University of Idaho

875 Perimeter Drive, Idaho 83844-1103

USA, Email: sdatta@uidaho.edu

Rae Young Kim

Department of Mathematics, Yeungnam University

280 Daehak-Ro, Gyeongsan, Gyeongbuk 38541

Republic of Korea, Email: rykim@ynu.ac.kr 